\title{
Systemwissenschaftliche Aspekte zur Tinnitus-Therapie
}

\author{
Uwe H. Ross \\ Praxis für Otorhinolaryngologie \& Psychotherapie, DE-Freiburg i. Br.
}

$D^{2}$ er Begriff Tinnitus (von lat. tinnire = klingeln) beschreibt eine komplexe Hörwahrnehmungsstörung durch Töne oder Geräusche, die - ohne äussere Schallquelle - im Ohr oder im Kopf lokalisiert wahrgenommen werden. In westlichen Industrienationen nehmen ca. 8\% der Bevölkerung Tinnitus permanent wahr, während $0,5-1 \%$ der Bevölkerung an diesem Symptom infolge Konzentrations- und Aufmerksamkeitsstörungen, Schlafstörungen und sozialer Isolation leiden [1,2]. Ob und wie sehr jemand unter Tinnitus leidet, hängt dabei nicht von Art der Ohrgeräusche (Lautheit, Frequenz, Klangfarbe) ab, sondern einzig von der subjektiven Bewertung und Verarbeitung des Signals. Diese wird in besonderem Masse durch psychosoziale Belastungen zum Zeitpunkt des Erstauftretens des Tinnitus bestimmt: Liegen aussergewöhnliche Belastungen, Ängstlichkeit, Lebensunzufriedenheit oder Schlafstörungen vor, ist eine nachfolgende Leidensentwicklung und Chronifizierung wahrscheinlicher als ohne diese Faktoren [3]. In der vorliegenden Arbeit werden zunächst gängige Therapieansätze bei störendem Tinnitus aus verschiedenen gesundheitswissenschaftlichen Perspektiven beleuchtet, um dann ein systemtheoretisches Symptomverständnis vorzustellen, welches das Symptom Tinnitus in seiner wechselnden Intensität als „Kompetenz“ des Organismus verstehbar, es im Sinne einer ressourcenorientierten Therapie nutzbar und für den Patienten als gesundheitsförderliches Feedback-Signal in Hinblick auf die Lebensgestaltung integrierbar macht.
Hintergrund: Anhaltend störender Tinnitus stellt in seiner Häufigkeit eine der grössten therapeutischen Herausforderungen dar, zumal übliche defizitorientierte Vorgehensweisen nach den mechanistischen Anschauungen der Biomedizin hier nicht dauerhaft greifen und eine Chronifizierung des Leidens begünstigen. Zielsetzung: Vorstellung systemwissenschaftlich fundierter Aspekte zur integrativen Tinnitus-Therapie, welche der Komplexität der Aufrechterhaltung der einseitig auditiven Fokussierung der Aufmerksamkeit gerecht wird. Methodik: Beleuchtung gängiger Ansätze der Tinnitus-Therapie aus verschiedenen gesundheitswissenschaftlichen Perspektiven und Darstellung eines Symptomverständnisses aus dem Blickwinkel der Systemtheorie, welche Tinnitus als Kompetenz des Organismus verstehbar und im Sinne einer ressourcenorientierten Therapie nutzbar macht. Ergebnisse und Schlussfolgerungen: Die Vorteile einer solchen Konzeption liegen in der positiven Neubewertung des Tinnitus-Signals durch den Betroffenen als gesundheitsförderndes FeedbackSignal. Ein solcher Bedeutungswandel beim Betroffenen führt zu einer nachweisbaren Abnahme des Belastungsgrades nach standardisierten Kriterien des Tinnitus-Fragebogens nach Goebel \& Hiller. Systemische Ansätze der modernen Erickson'schen Hypnose werden hier als sinnvolle und hilfreiche Verfahren angesehen, auf der Grundlage des gesundheitswissenschaftlichen Konzeptes der Salutogenese problemimmanente Kompetenzen des Betroffenen zu utilisieren und über die systematische Fokussierung von Aufmerksamkeit individuelle Gesundheitsressourcen zu mobilisieren. Mit der Meta-Konzeption moderner Systemtheorien lassen sich neben der modernen Hypnose auch andere regulationsmedizinische Therapie-Ansätze zur Mobilisierung von Gesundheitspotenzialen innerhalb eines ressourcenortientierten Therapie-Konzeptes sinnvoll integrieren.

Schlüsselwörter: Tinnitus, Systemtheorie, Salutogenese, Erickson'sche Hypnose

\section{Systems Science-Based Aspects of Tinnitus Therapy}

Background: Persistent and disturbing tinnitus is a major therapeutic challenge as its incidence is increasing and conventional approaches using mechanistic biomedical models are mainly ineffective in the long term and may induce development of a chronic condition. Objective: Presentation of a systems science-based approach of integrative tinnitus therapy which allows for the complexity of maintaining one-sided auditive focussing of attention. Methods: Discussion of conventional approaches to tinnitus therapy from various perspectives of health science with presentation of the symptom model from a systems theory's view point that regards tinnitus as a functional pattern that can be utilised as a competence of the organism in the sense of resource-orientated therapy. Results and conclusion: The advantages of this concept lies in the positive re-evaluation (reframing) of the increased tinnitus sensivity by the affected person as a health-promoting feedback signal of the organism related to stressful situations. The change in significance leads to a demonstrable decrease in tinnitus-related stress and tinnitus-related burdens verified by a standardised tinnitus questionnaire (TQ, German version after Goebel and Hiller). Systemic approaches of modern Ericksonian hypnosis are regarded as a reasonable procedure to help the affected person, using the concept of salutogenesis to exploit problem-inherent competences and to mobilise the individual's own health resources via guided focusing of the patient's attention. With the meta-concept of modern system theories, not only modern hypnosis but also other approaches of regulative medicine to mobilise health-giving potentials can be integrated into a resource-oriented therapeutic concept.

Key words: Tinnitus, Systems theory, salutogenesis, Ericksonian Hypnosis 


\section{Phasengerechte}

\section{Diagnostik und Therapie}

Nach der aktuellen Klassifikation ist je nach Persistenz akuter Tinnitus (Dauer: <3 Monate) von subakutem (Dauer: 3 bis <12 Monate) und chronischem Tinnitus (Dauer: >12 Monate) zu unterscheiden [4]. Diese willkürlich getroffene Klassifikation soll dem Arzt spezifische standardisierte Handlungsanweisungen hinsichtlich Diagnostik und Therapie geben. In der Akutphase ist daher eine audiologisch-neurootologische Diagnostik in Regie des HNOArztes indiziert, um etwaige Veränderungen auf der Ebene der Körperstruktur als auslösende Ursachen für Tinnitus zu erfassen, aus denen sich hilfreiche medikamentöse oder operative Behandlungsoptionen ergeben (Tab. 1). In seltenen Fällen liegt ein objektiver Tinnitus vor, der ggf. mit dem Stethoskop mithörbar ist und aus einer körpereigenen Schallquelle resultiert. Ursachen hierfür können Myoklonien der Mittelohr- oder Gaumenmuskulatur, Gefässanomalien oder -tumore sein. Zumeist handelt es sich jedoch um subjektiven (nicht-mithörbaren) Tinnitus, der hier nachfolgend behandelt werden soll.

In vielen Fällen kann eine auslösende oder somatisch angehbare Ursache für subjektiven Tinnitus nicht objektiviert, sondern im Einzelfall lediglich vermutet werden. In Deutschland wird akuter Tinnitus, der in 68-80\% der Fälle mit einer Hörminderung einher geht, als „Hörsturz“-Äquivalent angesehen [2] und nach dem wissenschaftlich heftig diskutierten Durchblutungsparadigma zumeist mit Rheologika unter Kortikoidzusatz behandelt (Übersicht in [5],[6]). Die Spontanremissionrate für die akute idiopathische Hörminderung (so genannter Hörsturz) ist allerdings hoch und wird je nach Studie mit 56$89 \%$ beziffert (Übersicht in [5]). Klare Wirksamkeitsbelege nach EBM-Kriterien für den Einsatz von Rheologika bzw. für Kortikoide, deren Einsatz bislang als internationaler „Gold-Standard“ in der Therapie der akuten idiopathischen Hörminderung gilt, liegen bis heute nicht vor, wie eine erst kürzlich erschiene Metaanlyse von 21 randomisierten, kontrollierten Studien

Tab. 1. Auslösende Ursachen subjektiver Ohrgeräusche

\begin{tabular}{|c|c|c|c|}
\hline Kochleär & Neural & Zentral & Andere \\
\hline $\begin{array}{l}\text { Akute Hörminderung (sog. "Hörsturz") } \\
\text { Akutes Lärmtrauma } \\
\text { Idiopathisch (ohne Hörminderung) } \\
\text { Chronische Lärmschwerhörigkeit } \\
\text { Endolymphhydrops (Menière-Trias) } \\
\text { Infektiös-toxische Innenohrschädigung } \\
\text { Ototoxische Medikamente } \\
\text { Otosklerose } \\
\text { Perilymphfistel } \\
\text { Commotio / Contusio labyrinthi } \\
\text { Chron.-progred. Innenohrschwerhörigkeit } \\
\text { Immunogene Innenohrschwerhörigkeit } \\
\text { Hereditäre Schwerhörigkeit }\end{array}$ & $\begin{array}{l}\text { Akustikusneurinom } \\
\text { Multiple Sklerose }\end{array}$ & $\begin{array}{l}\text { Tumore } \\
\text { Traumata (SHT) } \\
\text { Ischämien im Bereich } \\
\text { der zentralen Hörbahn }\end{array}$ & $\begin{array}{l}\text { HWS-Dysfunktion } \\
\text { Myoarthropathie des } \\
\text { Kiefergelenks }\end{array}$ \\
\hline
\end{tabular}

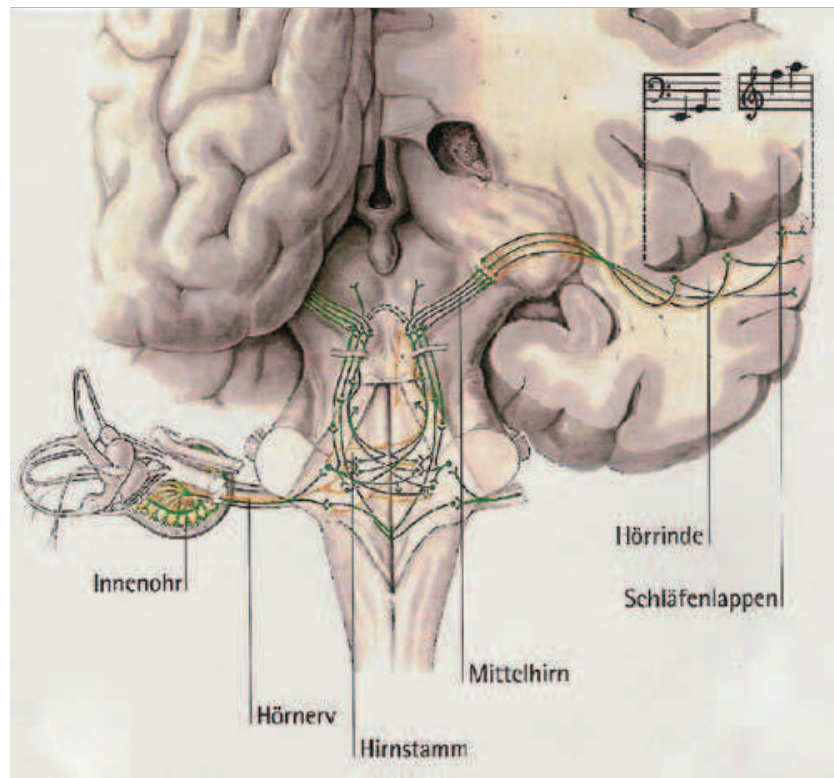

Durch auslösende Ursachen (s. Tab.1) wird eine erhöhte tinnitus-relevante neurale Aktivität innerhalb der zentralen Hörbahn getriggert. Das initia entstandene "Störsignal" wird einem von aussen kommenden Schallreiz gleichgesetzt und nach dem Prinzip der Mustererkennung bis zur Hörrinde durchgeschaltet. Eine negative affektiv-kognitive Konnotation unter Beteiligung des limbischen Systems sowie des präfrontalen Kortex führt über die aufmerksamkeitssteuernde Instanz im Mittelhirn (Formatio reticularis) zur Veränderung neuraler Filterprozesse im Hörsystem. Die Weiterleitung sonst unterschwelliger auditiver Impulse wird so, begleitet durch die Bildung tinnitus-relevanter neuronaler Netzwerke, begünstigt und stabilisiert. (Abb. modifiziert nach [41]).

Abb. 1. Hörbahn und Signalverarbeitung bei chonischem Tinnitus.

ergab [7]. Vor diesem Hintergrund kann diese Therapie auch bei Tinnitus nicht zweifelsfrei empfohlen werden und muss an die individuelle Situation des Betroffenen (Therapiewunsch, Leidensdruck, Vorhandensein einer akuten Hörminderung) angepasst werden. Häufig werden auch phytotherapeutische Ginkgo-biloba-Präparate bei Tinnitus eingesetzt, denen eine rheologische und antioxidative Wirkung zugeschrieben wird. Eine aktuelle Metaanalyse konnte eine Wirksamkeit bei Tinnitus nach EBM-Kriterien allerdings nicht nachweisen [8].

Bereits im Rahmen der Akutphase bietet sich ebenfalls eine gnathologi- sche und eine manualtherapeutische Diagnostik an, um Tinnitus stabilisierende Einflüsse von Seiten des Kiefergelenks (Bruxismus, Myoarthropathie) oder der HWS zu erfassen und ggf. zu behandeln. Wenn sich auch hieraus keine zielführenden Behandlungsoptionen ergeben, den Tinnitus zu beseitigen, zielen therapeutische Bemühungen insbesondere bei subakutem und chronischem Tinnitus darauf ab, die negative kognitiv-affektive Bewertung des Tinnitus-Signals zu verhindern bzw. zu relativieren. Denn diese verstärkt die Penetranz des Tinnitus, unterhält dessen Chronifizierung [3] und führt mit der Zeit nachweislich $\mathrm{zu}$ 
einer tinnitus-relvanten, neuronalen Aktivierung und Umstrukturierung im auditorischen Kortex [9,10,11]. Eine Reduktion von psycho-sozialen Belastungsfaktoren und das Erlernen von Entspannungstechniken ist daher bereits in der Akutphase hilfreich.

\section{Tinnitus-Retraining-Therapie}

Aus dem anglo-amerikanischen Raum stammend hat das ambulante Konzept der Tinnitus-Retraining-Therapie (TRT) auf der Basis eines neurophysiologischen Tinnitus-Modells (Abb. 1, [12]) seit den 1990er Jahren starke Verbreitung erfahren. Es sieht bei störendem, subakutem und chronischem Tinnitus die integrative Zusammenarbeit von HNO-Heilkunde, Psychotherapie und Hörgeräte-Akustik mit der Verordnung von Rauschgeräten vor. Eine neben dem Tinnitus bestehende, kommunikativ relevante Schwerhörigkeit sollte - wenn nötig - mit Hörgeräten ausgeglichen werden, um tinnitusrelvante zentralnervöse Filterprozesse günstig zu beeinflussen und über eine Teilmaskierung des Tinnitus durch die Verstärkung der Aussengeräusche eine Entlastung des Betroffenen $\mathrm{zu}$ erreichen. Die TRT zielt auf die Habituation (Gewöhnung) des Ohrgeräusches. Je nach Belastungsgrad sind hierzu ausser einem intensiven HNO-ärztlichen Beratungsgespräch („direktive Counceling“) auch die Vermittlung von Entspannungstechniken (Progressive Muskelrelaxation, Autogenes Training, Biofeedback), verhaltenstherapeutische Verfahren zur DeFokussierung und Stressbewältigung oder eine Psychotherapie sinnvoll [4]. Dieses ambulante, integrative Konzept der TRT hat sich inzwischen als effektiv erwiesen [13]. Die mit dem standardisierten Tinnitusfragebogen (TF, [14]) evaluierten Therapie-Effekte der ambulanten TRT bei chronischem Tinnitus mit einer durchschnittlichen Therapiedauer von 1-1,5 Jahren zeigen eine Abnahme des TF-Scores von durchschnittlich 14,1 Punkten. Diese Effekte sind denen einer vergleichsweise kürzeren, stationären TinnitusTherapie von 6-12 Wochen [15,16] bzw. 4 Wochen in einer eigenen, kürz- lich erschienenen Wirksamkeitsstudie mit 393 Patienten vergleichbar [17, 18].

\section{Gesundheitswissenschaft- liche Sichtweisen und ihre therapeutischen Implikationen}

\section{Das Pathogenese-Modell}

Traditionell ist in der Medizin ein pathogenetisches, biomedizinisches Symptomverständnis vorherrschend, das - der Leitfrage folgend: Was macht uns krank? - eine Störung durch lokalisierte somatische Defekte und als sukkzessive Abfolge von Kausalereignissen erklärbar und behandelbar macht. Dieses Modell hat bekanntermassen $\mathrm{zu}$ immensen Fortschritten der Biomedizin geführt, wie bei der Behandlung von mechanischen Traumata, Infektionen, Stoffwechselerkrankungen u.a. [19]. Dies trifft auch für viele der in Tab. 1 genannten auslösenden Tinnitus-Ursachen zu.

Seit Mitte der 1990er Jahre wurden bei chronischem Tinnitus veränderte Funktionsweisen in verschiedenen Regionen des ZNS nachweisbar: Aktivitätserhöhungen im primären und sekundären auditorischen Kortex, im präfrontalen Kortex, im Thalamus, im limbischen System [9,11] sowie Veränderungen der tonotopischen Karten des auditorischen Kortex [10]. Auch liess sich bei Tinnitus-Betroffenen über stereotaktisch applizierte EEG-Mikroelektroden eine dysfunktionale Prozessierung auditorischer Signale im medialen Thalamus im Sinne einer thalamokortikalen Dysrhythmie nachweisen, die als Folge einer peripheren sensorischen Deafferenzierung bei Innenohrschwerhörigkeit angesehen wird [20].

Dem Pathogenese-Modell folgend zielen einige neuere Therapieansätze sodann darauf ab, störenden chronischen Tinnitus auf neurophysiologischer Ebene der zentralen Hörbahn zu behandeln: Die 5-tägige, repetitive transkranielle Magnetstimulation (rTMS) über dem temporalen Kortex führte bei Tinnitus-Betroffenen für einige Minuten zur passageren Verminderung der Tinnitus-Wahrnehmung. Die berichtete Abnahme der Tinnitus-Belastung im TF und die Nachhaltigkeit der Effekte waren allerdings vergleichsweise gering $[21,22]$, so dass die TMS gegenwärtig nicht als klinisch einsetzbare Methode bei Tinnitus empfohlen werden kann. Ein anderer Ansatz zielt über eine stereotaktische mediale Thalamotomie auf eine Veränderung der dysfunktionalen neuronalen Aktivität bei chronischem Tinnitus auf Thalamus-Ebene, mit der bei einem Teil der vergleichsweise kleinen Patientengruppe eine 50-100\%ige Erleichterung erreicht wurde [20]. Die Anwendung bei störendem Tinnitus wird von den Autoren allerdings dadurch eingeschränkt, dass ein existenter mentaler, u.a. emotionaler Einfluss bei der thalamokortikalen Dysrhythmie über das limbische System mit dieser Methode offenbar nicht reduziert werden und im Einzelfall eine begleitende Psychotherapie erforderlich machen kann [23]. Es ist jedoch gerade die kognitive und emotionale Betroffenheit, die - z.B. mit dem TF [14] nach standardisierten Kriterien erfasst - das Ausmass des Leidens und damit die Behandlungsnotwendigkeit bestimmt.

Die Anwendung biomedizinisch-pathogenetischer Behandlungsansätze bei anhaltend störendem Tinnitus (pharmakologisch, neurophysiologisch, chirurgisch) ist auch aus anderen Gründen nicht ganz unproblematisch: Auf Grund ihrer Somatisierungstendenz sind Tinnitus-Betroffene sehr empfänglich für biomedizinische Behandlungsmodelle und -ansätze, da die Aussicht auf Beseitigung des Symptoms auf somatischer Ebene eine rasche Linderung des Leidens und Entlastung „von aussen“ verspricht und dies auch der traditionellen Rollenerwartung des Patienten an den Arzt als „Reparateur“ des Körpers entspricht. Je länger störender Tinnitus jedoch anhält, desto mehr gewinnen - wenn nicht schon primär vorhanden - neben den auslösenden Ursachen auf biologischer Ebene (Tab. 1) aufrechterhaltende Ursachen psychosozialer Art (emotional-kognitive Bewertung, Konditionierungseffekte, Änderung sozialer Beziehungen u.a.) im Rahmen eines multifaktoriellen, biopsycho-sozialen Zusammenspiels an Einfluss. Mit jedem frustrierenden Therapieversuch, dieses komplexe Geschehen auf Körperebene beseiti- 
gen zu wollen, wird einer Verstärkung und Chronifizierung des Leidens daher eher Vorschub geleistet.

In der Therapie von anhaltend störendem Tinnitus geht es vielmehr darum, den Betroffenen in einer kooperativen Arbeitsbeziehung zur aktiven Mitarbeit im Umgang mit dem Tinnitus sowie der Neuausrichtung seiner Lebensführung anzuregen [4].

\section{Grenzen des Pathogenese-Modells}

Wenngleich einem bio-psycho-sozialen Modell [24] folgend, arbeiten sowohl die ambulante TRT als auch übliche stationäre Therapiekonzepte zumeist aus der Perspektive des defizitorientierten Pathogenese-Modells der Medizin. Dieses gebräuchliche Krankheitsmodell impliziert eine Haltung (bei Patient und Arzt), die das Symptom und die Art seiner Verarbeitung einseitig als Defekt und damit als Inkompetenz des Systems („Störung“) definiert, die es zu „bewältigen“ gilt. Diese Strategie der TRT erweist sich bei ausreichender Willenskraft zunächst als hilfreich, stösst aber bald auf ihre Grenzen. Denn nicht selten kämpfen die Betroffenen auf diese Weise ständig gegen „etwas“ in sich selbst, das offenbar nicht vollständig ihrem Willen unterliegt, im dem Sinne: „Ich will ja schon meine Aufmerksamkeit woanders hinlenken, aber es geschieht ganz unwillkürlich, dass ich auf den Tinnitus hören muss.“

Nach den Ergebnissen der modernen Hirnforschung müssen wir davon ausgehen, dass ein Grossteil unserer körperlichen und seelisch-geistigen

Tab. 2. Merkmale von Tinnitus-Betroffenen aus pathogenetischer und salutogenetischer Sicht und deren therapeutische Nutzungsmöglichkeiten [17]

\begin{tabular}{|c|c|c|}
\hline \multicolumn{3}{|c|}{ Perspektive } \\
\hline pathogenetisch & salutogenetisch & Therapeutische Nutzbarkeit \\
\hline Neigung zur Intellektualisierung & Hohe intellektuelle Auffassungsgabe & Gesundheitsbildung \\
\hline $\begin{array}{l}\text { Neigung zur Selbstbeobachtung } \\
\text { Überempfindlichkeit }\end{array}$ & $\begin{array}{l}\text { Wahrnehmungsfähigkeit des inneren } \\
\text { Erlebens und Sensibilität für sich selbst }\end{array}$ & $\begin{array}{l}\text { Imaginative Verfahren, Hypnose } \\
\text { Ausbau der propriorezeptiven } \\
\text { Wahrnehmungskompetenzen }\end{array}$ \\
\hline $\begin{array}{l}\text { Tinnitus ist eine nutzlose } \\
\text { Wahrnehmungsstörung }\end{array}$ & $\begin{array}{c}\text { Tinnitus besitzt } \\
\text { kontextabhängigen Signal-Charakter } \\
\text { und semantische Information }\end{array}$ & Klärung der Sinnhaftigkeit \\
\hline
\end{tabular}

Aktivitäten von unwillkürlich-unbewussten Prozessen geprägt ist (z.B. [25]) - so auch die anhaltend störende Tinnitus-Wahrnehmung (siehe [12]). In dieser Situation wären Ansätze hilfreich, die zusätzlich eine positive Einflussnahme auf unwillkürlicher Ebene der impliziten Informationsverarbeitung und bestenfalls eine Kooperation mit dem Symptom Tinnitus ermöglichen. Dazu bedarf es eines modifizierten Symptomverständnisses auf Seiten des Arztes wie des Patienten. Der Arzt sollte also, gewissermassen aus einer Meta-Position, eine andere als die pathogenetische „Brille“ finden, mit der er seine Patienten und das Symptom Tinnitus betrachtet.

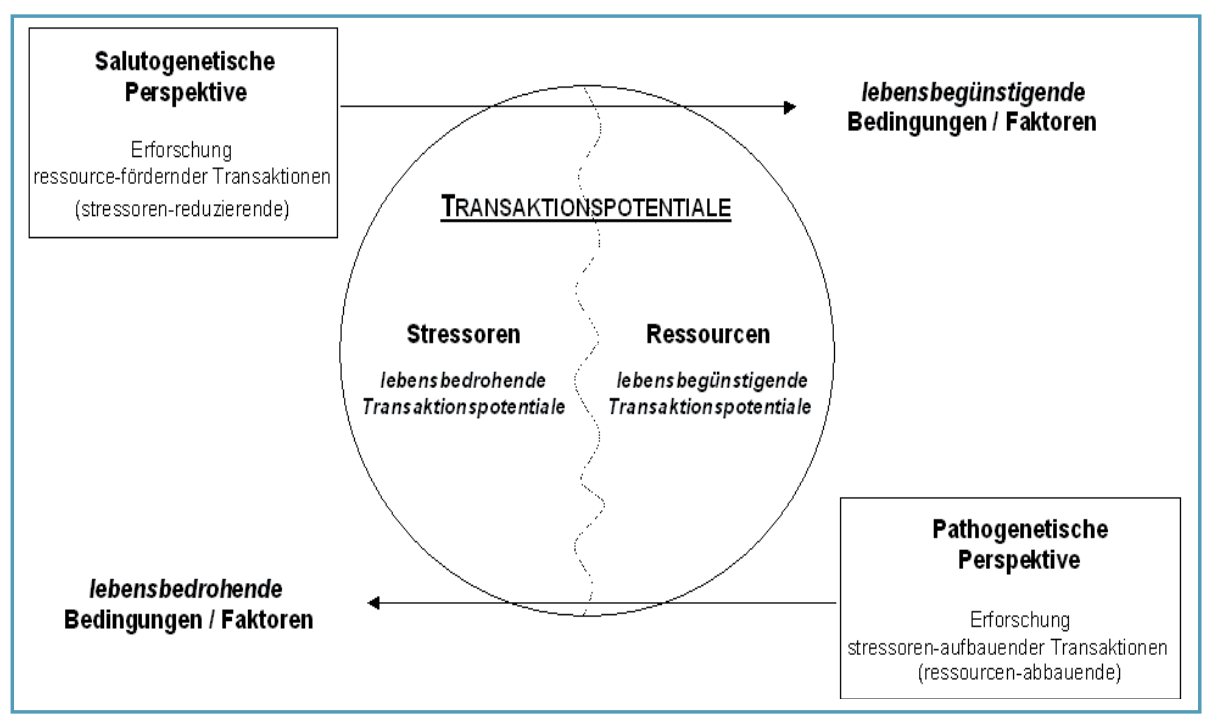

Abb. 2. Pathogenetische und salutogenetische Perspektiven (nach [40]).

\section{Das Salutogenese-Modell}

Die problem- und krankheitsorientierte Sichtweise nach dem pathogenetischen Modell mit dem Prinzip der Vermeidung bzw. Beseitigung von Risikofaktoren und Krankheitsursachen (,weg von“-Orientierung) ist heute immer noch vorherrschend ([19], Abb. 2). Bei anhaltend störendem Tinnitus ist dieses Modell wenig zweckmässig, da jede Bemühung, das Symptom zu beseitigen oder seine Wahrnehmung zu verdrängen, zu seiner Aufwertung im Sinne einer problemorientierten Aufmerksamkeitsfokussierung führt. Demgegenüber ergibt sich aus dem ressourcen-orientierten, gesundheitswissenschaftlichen Modell der Salutogenese (lat. salus $=$ gesund, griech. genesis $=$ Entstehung) des israelischen Medizinsoziologen AARON ANTONOvsky [26] eine Sichtweise, aus welcher die kontextabhängig unterschiedliche Symptomausprägung und die Art der Verarbeitung durch den Patienten als Systemkompetenz gewürdigt und für die Therapie genutzt werden kann (Tab. 2).

Innerhalb eines Kontinuums von Gesundheit und Krankheit stehen insbesondere Bedingungen und Wirkfaktoren, die Gesundheit in Anwesenheit von Stressoren schützen und aufrechterhalten, im Blickpunkt des Salutogenese-Konzeptes. Gesundheit wird hierin positiv konzeptualisiert, denn die Kernfrage „Was hält uns gesund?“ (statt: Was macht uns krank? im Pa- 
thogenese-Modell) führt $\mathrm{zu}$ Faktoren, die nach AnTONOvSKY bei der Mobilisierung gesundheitsrelevanter Ressourcen entscheidend sind, nämlich die Verstehbarkeit, Handhabbarkeit und Bedeutsamkeit von Ereignissen im Leben [26]. Aus diesen Faktoren ergibt sich eine individuelle Grundhaltung der Stimmigkeit (Kohärenzsinn bzw. -erleben [27]), die erklärt, wie Menschen trotz vergleichbarer gesundheitswidriger Umstände in sehr unterschiedlichem Masse in der Lage sind, vorhandene Ressourcen für den Erhalt ihrer Gesundheit zu nutzen [19,26]. Eine wichtige Implikation, die diese ressourcen-orientierte, gesundheitswissenschaftliche Sichtweise bei störendem Tinnitus hat, ist die zunächst paradox klingende Frage, wie Tinnitus bzw. seine störende und regelmässig beobachtbare kontextabhängige Intensivierung, selbst als Ressource vom Betroffenen genutzt werden kann.

\section{Systemwissenschaftliches Symptomverständnis}

Nach Konzepten zur Selbstorganisation von lebenden Systemen, wie der Autopoiese [25,28,29], der Synergetik [30] sowie der Informationstheorie und Biosemiotik [31] kann anhaltend stö-
Tab. 3. Häufige implizite Bedürfnisse bei störendem Tinnitus

- Dissoziation von „problematischen“ Affekten
- Grenzziehung nach außen, innerer Abstand
- innerer Freiraum
- Selbstwahrnehmung
- Entlastung
- Sicherheit
- (Selbst-) Zuwendung

render Tinnitus als dynamisches, biopsycho-soziales Funktionsmuster aufgefasst werden, über welches bestimmte System-Erfordernisse bzw. Bedürfnisse des Betroffenen auf der Ebene impliziter Informationsverarbeitungsprozesse, d.h., unwillkürlich und autonom, zum Ausdruck kommen (Tab. 3). Das in Hinblick auf ein willentlich angestrebtes Ziel (z.B. Leistungsfähigkeit, Rollen- und Pflichterfüllung) problematisch erlebte Symptommuster „Tinnitus“ steht somit eigentlich im Dienste des Systemerhalts. Das kontextabhängig unterschiedlich stark wahrgenommene Symptom stellt daher den aktuell bestmöglichen Lösungsversuch für den psycho-physischen Organismus dar, implizite, nicht gelebte Bedürfnisse unwillkürlich-autonom auszudrücken und so auf Belastungen physischer und

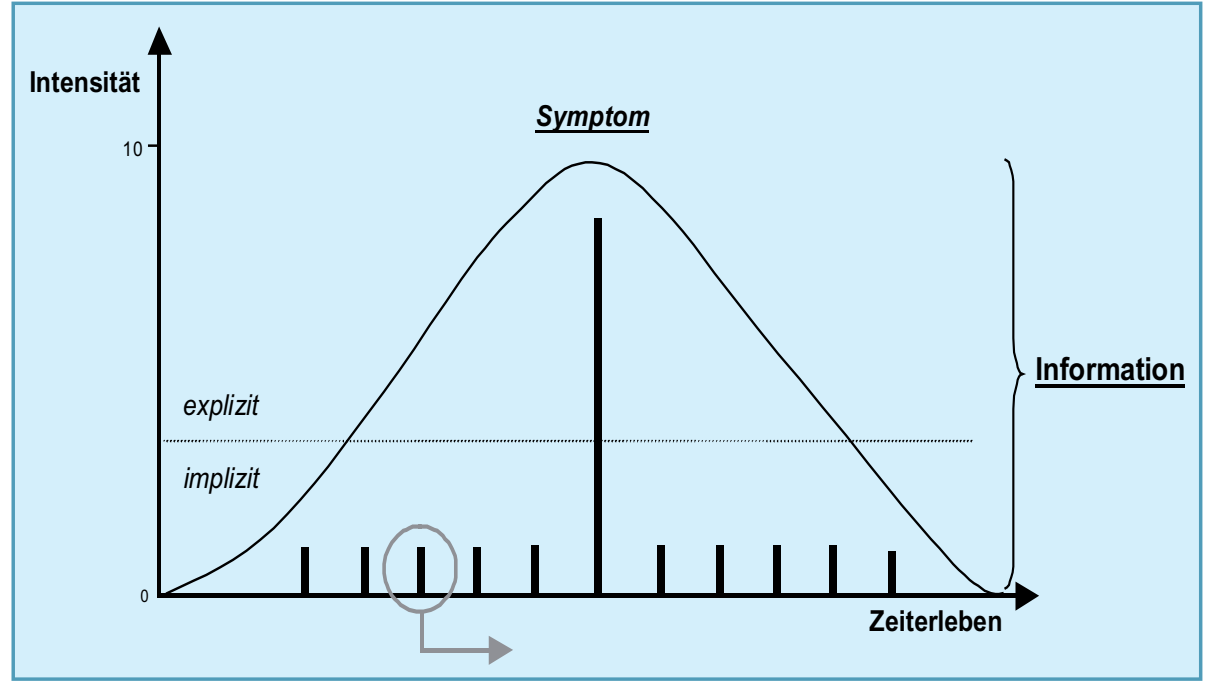

Abb. 3. Schematisierte Darstellung der subjektiv wahrgenommenen Symptomausprägung in Abhängigkeit von Veränderungen der Kontextbedingungen entlang der Zeit.

Die kontextabhängig wechselnde Ausprägung des Symptoms kann als "Symptom-Trance" [34, 35] verstanden werden, welche aus einer zeitlichen Abfolge verschiedener, zumeist impliziter Erlebniselemente aus Wahrnehmungen, Affekten, Gedanken und Verhalten besteht und in einer intensiven bewussten Symptom-Wahrnehmung gipfelt. Die Veränderung strategischer Schlüsselelemente ist geeignet, eine Unterbrechung des bisherigen Musters im Erleben des Betroffenen zu bewirken psycho-sozialer Kontextbedingungen zu reagieren. Das Auftreten von Symptomen wie Tinnitus resultiert dabei aus einem Übersetzungsproblem zwischen den Zeichenebenen verschiedener Integrationsebenen [32]. Um herauszufinden, welchen Informationswert (Tab. 3) die meist kontextabhängig wechselnde Penetranz des Tinnitus im Einzelfall hat, bieten sich systemische Ansätze der Erickson'schen Hypnose an, da sie geeignet sind, Zeichen insbesondere auf der Ebene impliziter informationsverarbeitender Prozesse $\mathrm{zu}$ entschlüsseln und gesundheitsförderliche Prozesse auf unwillkürlicher Ebene zu aktivieren. Nach moderner Begriffsfassung wird Hypnose als interaktioneller Prozess einer systematischen Fokussierung von Aufmerksamkeit verstanden, um eine Trance zu induzieren. Trance ist ein veränderter Bewusstseinszustand mit dem Vorherrschen unwillkürlicher Prozesse, ein ganz natürliches und alltägliches Phänomen, das auch beim vertieften Lesen eines Buches oder Schauen eines Kinofilms auftritt [33,34]. Die therapeutische Trance stellt somit eine Sonderform dar, die insbesondere durch Entspannung, Ressourcen- und Lösungsbewusstsein des Klienten geprägt ist.

\section{Symptomverständnis und hynotherapeutisches Vorgehen bei Tinnitus}

Im Sinne der modernen Hypnose wird das „symptomatische“ bio-psychosoziale Funktionsmuster Tinnitus als „Symptom-Trance“ [34,35] angesehen, d.h. als Vorherrschen unwillkürlicher, allerdings unerwünschter Prozesse, die aus der verdichteten Abfolge miteinander assoziierter Elemente aus Wahrnehmung, Affekten, Gedanken und Verhalten bestehen und in einer bewussten Symptomwahrnehmung gipfeln (Abb. 3).

Aus der hypnotherapeutischen Beleuchtung der zumeist unbewussten, unwillkürlichen Abfolge symptomstabilisierender Elemente im subjektiven Erleben ergeben sich zahlreiche therapeutische Optionen zur gesundheitsförderlichen Umgestaltung des bishe- 
rigen, problematisch erlebten Funktionsmusters auf unwillkürlicher Ebene mittels Hypnose (Übersicht in [36,37]). Hierbei wird dem Patienten zunächst die Bedeutung des Symptoms als Systemkompetenz deutlich. Des Weiteren ergibt sich ihm hieraus ein Gesundheitsziel, welches meist einem bedürfnisorientierten Meta-Ziel entspricht (z.B. innere Ruhe und Genussfähigkeit entwickeln), hinter dem vordergründigen Ziel, den Tinnitus nicht mehr wahrnehmen zu wollen. Bei der weiteren Entwicklung des Ziel-Zustandes werden sowohl zu aktivierende Ressourcen des Betroffenen (kraftvolle innere Zustände, Kompetenzen) sowie gesundheitseinschränkende Glaubenssätze (z.B. „Wenn der Tinnitus anhält, finde ich keine Ruhe mehr.") als auch die Wechselwirkungen mit dem sozialen Kontext thematisiert (Abb. 4).

Im Rahmen einer ressourcen-orientierten Tinnitus-Therapie [18,37] sind Ansätze der moderenen Hypnose geeignet, über die systematische Fokussierung von Aufmerksamkeit innere Ressourcen des Betroffenen in Hinblick auf das individuelle Gesundheitsziel zu mobilisieren. Phasen, in denen das Tinnitus-Signal in den Hintergrund tritt oder in der Wahrnehmung gänzlich ausbleibt, gelten als bestätigendes Feedback für eine bedürfnisgerechte und gesundheitsfördernde Lebensweise. In der Umgebung des privaten und beruflichen Alltags tragen die gewonnenen Informationen und Erfahrungen dazu bei, dem TinnitusSignal eine zielgerichtete, positive Bedeutung zu geben, so dass es zukünftig als Erinnerungshilfe für die zu integrierende Ressource bzw. das beachtenswerte Bedürfnis und damit als „Kooperationspartner“ in Sachen Gesundheit nutzbar ist (Fallbeispiele hierzu in [37]).

\section{Wirkunsweise der Hypnose auf neurophysiologischer Ebene}

Inzwischen lassen sich die Wirkungen von Hypnose mittels bildgebender Verfahren, wie PET und fMRI, auf neurobiologischer Ebene nachweisen. Hypnose führt demnach $\mathrm{zu}$ neuronalen

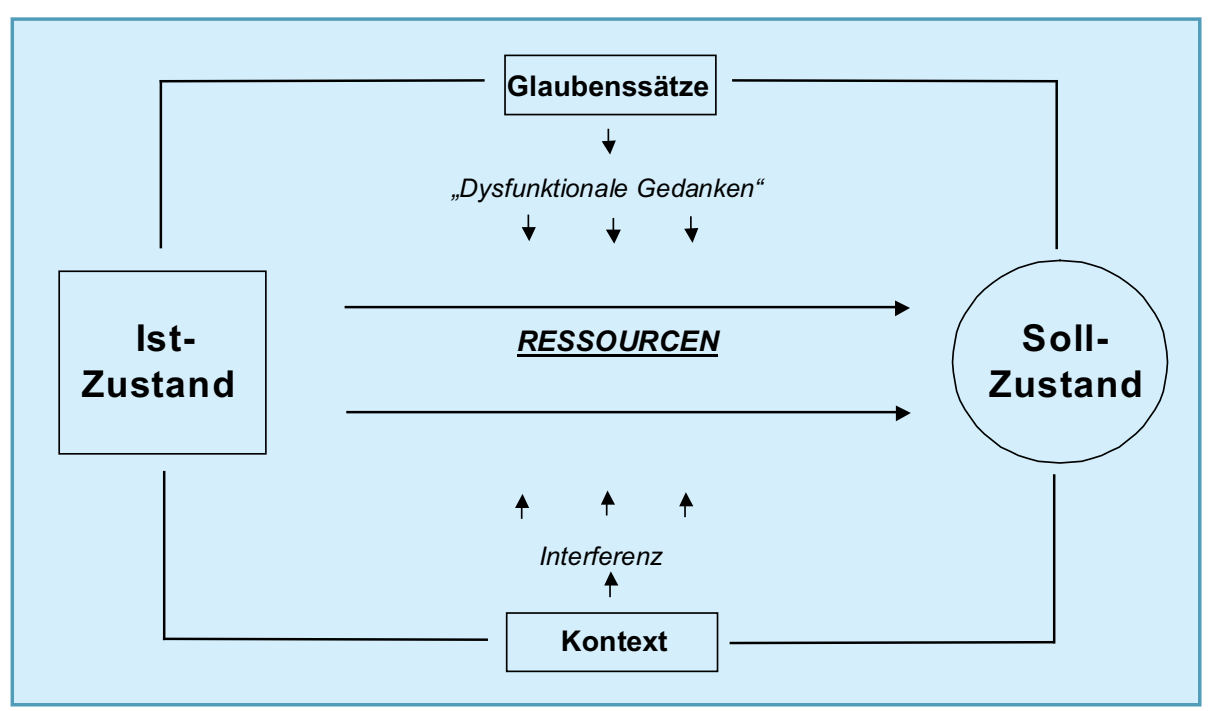

Abb. 4. Bewegungen vom Ist-Zustand (subjektive Befindlichkeit „krank”) zum Soll-Zustand (Gesundheitsziel) im mehrdimensionalen Krankheits-Gesundheits-Kontinuum [17].

Aktivitätsänderungen insbesondere im vorderen cingulären Cortex, in Thalamus und Hirnstamm, daneben im präfrontalen Cortex und im occipitalen Cortex [38]. Eine mittels Hypnose induzierte Veränderung des affektiven und sensorischen Schmerzerlebens geht nachweislich mit neuronalen Aktivitätsänderungen im vorderen cingulären Cortex bzw. im somatosensorischen Cortex einher [38]. Die Befunde belegen die Möglichkeit der verbalen, hypnotisch induzierten Modulation der Verarbeitung sensorischer Erfahrungen in Verbindung mit Veränderungen hirnphysiologischer Vorgänge über Top-down-Prozesse [39].

\section{Zusammenfassung und Schlussfolgerung}

Die Verbreitung des ambulanten Tinnitus-Retraining-Therapie (TRT)-Konzeptes im deutschsprachigen Raum ist mit einer interdisziplinären Zusammenarbeit von HNO-Heilkunde, Psychotherapie und Hörgeräteakustik und deutlich verbesserter Versorgung der Betroffenen verbunden. Doch ebenso wie dieses ambulante gehen auch stationäre Therapie-Konzepte von einer pathogenetischen Sicht aus, die das Symptom und dessen Verarbeitungsweise durch den Patienten einseitig als defizitär abwertet. Eine systemwissenschaftlich fundierte salutogenetische, d.h. ressourcen-orientierte Sichtweise erlaubt dagegen, bestimmte Merkmale und Strategien des Patienten, die bislang zur Aufrechterhaltung des symptomatischen Funktionsmusters beitrugen, als Ressourcen zu nutzen und ermöglicht damit eine wertschätzende Kooperation in der Therapie. Als praktische Umsetzung des gesundheitswissenschaftlichen Konzeptes der Salutogenese von AnTonovsky [26] werden moderne Ansätze der Erickson'schen Hypnose [34,36] angesehen, zumal sie zahlreiche Analogien ihrer Grundprämissen aufweisen wie beispielsweise das grundsätzliche Vorhandensein von Ressource-Potenzial zur gesundheitsfördernden Selbstorganisation beim Patienten [17].

Ein solches systemwissenschaftlich begründetes Therapiekonzept mit schwerpunktmässiger Anwendung moderner Ansätze der Erickson'schen Hypnose hat sich im stationären Kontext in einer kontrollierten Studie mit 393 Patienten als überaus wirksam erwiesen, die Tinnitusbelastung innerhalb eines vergleichsweise kurzen Behandlungszeitraum von 28 Tagen signifikant zu senken [18].

Wie dargestellt, erfordert die Komplexität der multifaktoriellen Zusammenhänge bei störendem Tinnitus zu ihrem Verständnis mehrere Modelle, die jeweils unterschiedliche Implikationen haben. Der Wert und Nutzen dieser Modelle bemessen sich daran, 
inwieweit sie dem Ziel und der Aufgabe des Arztes dienen. So stellt sich für den Arzt die Frage, welches der Modelle (bio-medizinisch, bio-psychosozial, pathogenetisch, salutogenetisch) in Hinblick auf gewünschte Veränderungen zieldienlich ist. Der Patient ist dabei als Kooperationspartner anzusehen, der vor dem Hintergrund eigener Störungstheorien mit entscheiden sollte, nach welchem Modell er behandelt, beraten oder begleitet werden will.

In diesem Sinne liefert die vorliegende Arbeit einen Beitrag zur anstehenden Entwicklung eines bio-psychosozialen Tinnitus-Modells, welches aus den genannten Gründen vorzugsweise auf verdeckte Ressourcen bzw. Kompetenzen des Systems ausgerichtet sein sollte.

Praxis und Klinik mit ihren kraftvollen, spezifischen Denk-, Sprachund Handlungsstrukturen in Diagnostik und Therapie haben zusätzlich weit reichende Nutzungsmöglichkeiten als kontinuierliche, kommunikative Induktionsysteme zur Initiierung salutogenetischer Prozesse über die systematische ressource- und lösungsorientierte Fokussierung der Aufmerksamkeit des Patienten. Erforderlich hierfür ist eine ressourcen-orientierte Kommunikationskultur, die neben der jeweiligen Störung oder Einschränkung die gesunden Anteile des Patienten im den Blick behält und diese aktiviert.

\section{Literatur}

1. Coles, R.R. Epidemiology of Tinnitus (I) J. Laryngol. Otol. 1984;9 (suppl):7-15.

2. Lenarz, T. Diagnostik und Therapie des Tinnitus. Laryngo-Rhino-Otol 1998;77:54-60.

3. Olderog, M., Langenbach, M., Michel, O., Brusis, T., Köhle, K. Prädikatoren und Mechanismen der ausbleibenden Tinnitus-Toleranzentwicklung - eine Längsschnittstudie. LaryngoRhino-Otol 2004;83:5-13.

4. Deutsche Gesellschaft für Hals-Nasen-OhrenHeilkunde, Kopf- und Hals-Chirurgie. Leitlinie Tinnitus. HNO 1999;47:14-18.

5. Plontke, S. Gestörtes Hören. Konservative Verfahren. Laryngo-Rhino-Otol 2005;84:S1-36.

6. Deutsche Gesellschaft für Hals-Nasen-OhrenHeilkunde, Kopf- und Hals-Chirurgie. Leitlinie Hörsturz: Therapie. Allgemeine Bemerkungen. AWMF online 2004, http://leitlinien.net

7. Conlin, A.E., Parnes, L.S. Treatment of sudden hearing loss: I. A sytematic review. Arch Otolaryngol Head Neck Surg 2007:133:573- 581.

8. Hilton, M., Stuart, E. Ginkgo biloba for tinnitus. Cochrane Database Syst Rev 2004;(2): 2CD003852.

9. Arnold, W., Bartenstein, P., Oestreicher, E., Römer, W., Schwaiger, M. Focal metabolic activation in the predominant left auditory Cortex in patients suffering from tinnitus: a PET study with [18F] deoxyglucose. ORL 1996;58: 195-199.

10. Mühlnickel, W., Elbert, T., Taub, E., Flor, H. Reorganisation of auditory cortex in tinnitus. Proc Natl Acad Sci 1998;95:10340-10343.

11. Lockwood, A.H., Salvi, R.J., Coad, M.L. Towsley, M.L., Wack, D.S., Murphy, B.W. The functional anatomy of Tinnitus. Evidence for limbic system links and neural plasticity. Neurology 1998, 50:114-120.

12. Jastreboff, P.J., Hazell, J.W.P. A neurophysiological approach to tinnitus: clinical applications. Br J Audiol 1993;27:7-17.

13. Biesinger, E., Heiden, C., Greimel, V. Lendle, T., Höing, R., Albegger, K. Strategien in der ambulanten Behandlung des Tinnitus. HNO 1998;46:157-169.

14. Goebel, G. Hiller, W. Tinnitus-Fragebogen (TF). Ein Instrument zur Erfassung von Belastung und Schweregrad bei Tinnitus (Manual). Hogrefe, Göttingen, 1998.

15. Goebel, G. Fortschritte bei der verhaltensmedizinischen Diagnostik und Behandlung quälender chronischer Ohrgeräusche. Otolaryngol Nova 1995;5:178-189.

16. Hesse, G., Rienhoff, N.K., Nelting, M., Laubert, A. Ergebnisse stationärer Therapie bei Patienten mit chronisch komplexem Tinnitus. Laryngo-Rhino-Otol 2001;80:503-508.

17. Ross, U.H. Die praktische Umsetzung des Salutogenese-Konzeptes bei chronischem Tinnitus als systemischer Hörwahrnehmungsstörung. In: Wydler, H., Kolip, P., Abel, Th. (Hrsg) Salutogenese und Kohärenzgefühl. Grundlagen, Empirie und Praxis eines gesundheitswissenschaftlichen Konzepts. Juventa, Weinheim, München 2002, S. 161-171.

18. Ross U.H., Lange O., Unterrainer J., Laszig R. Ericksonian hypnosis in tinnitus therapy: effects of a 28-day inpatient multimodal treatment concept measured by Tinnitus-Questionnaire and Health Survey SF-36. Eur Arch Otorhinolaryngol 2007;264:483-488.

19. Bengel, J., Strittmatter, R., Willmann, H. Was erhält Menschen gesund? - Antonovskys Modell der Salutogenese - Diskussionsstand und Stellenwert. In: Bundeszentrale für gesundheitliche Aufklärung (BzgA) (Hrsg), Forschung und Praxis der Gesundheitsförderung Bd. 6. Köln, BzgA, 1998.

20. Jeanmonod, D., Magnin, M., Morel, A. Lowthreshold calcium spike bursts in the human thalamus. Common physiopathology for sensory, motor and limbic positive symptoms. sory, motor and limbic
Brain 1996; 119:363-375.

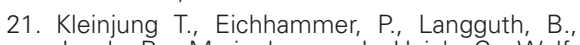
Jacob, P., Marienhagen, J., Hajak, G., Wolf, S.R., Strutz, J. Long-term effects of repetive transkranial magnetic stimulation (rTMS) in patients with chronic tinnitus. Otolaryngol Head Neck Surg 2005;132:566-569.

22. Plewnia, C., Reimold, M., Najib, A., Reischl, G., Plontke, S.K., Gerloff, C. Moderate therapeutic efficacy of positron emission tomography-navigated repetitive transcranial magnetic stimulation for chronic tinnitus: a randomised, controlled pilot study. J Neurol Neurosurg Psychiatry. 2007;78:152-156.

23. Jeanmonod, D., Magnin, M., Morel, Siegemund, M., Cancro, A., Lanz, M., Llinás, R., Ribary, U. Kronberg, E. Śchulman, J., Zonenshyn, M. Thalamocortical dysrhythmia II. Clinical and surgical aspects. Thalamus \& Rel Syst 2001; 1:245-254.

24. Engel, G.L. The need for a new medical model: A challenge for biomedicine. Science 1977; 196:129-136.

25. Roth, G. Selbstorganisation - Selbsterhaltung - Selbstreferentialität: Prinzipien der Organisation der Lebewesen und ihre Folgen für die Beziehung zwischen Organismus und Umwelt. In: Dress, A., Hendrichs, H., Küppers, G. (Hrsg): Selbstorganisation. Die Entstehung von Ordnung in Natur und Gesellschaft. Piper, München 1986, S.149-180.
26. Antonovsky, A. Salutogenese: Zur Entmystifizierung von Gesundheit. Dtsch. erweiterte Herausgabe von A. Franke. Dgvt-Verl., Tübingen, 1997.

27. Rimann, M., Udris, I. „Kohärenzerleben“ (Sense of Coherence): Zentraler Bestandteil von Gesundheit oder Gesundheitsressource. In: Schüffel, W. Brucks, U., Johnen, K., Köllner V., Lamprecht, F., Schnyder, U. (Hrsg) Handbuch der Salutogenese: Konzept und Praxis. Ullstein Medical, Wiesbaden1998, S. 351-373.

28. Maturana, H.R. Erkennen: Die Organisation und Verkörperung von Wirklichkeit. Ausgewählte Arbeiten zur biologischen Epistemologie. Vieweg, Braunschweig, Wiesbaden, 1982

29. Varela, F.J. Autonomie und Autopoiese. In: Schmidt, S.J. (Hrsg) Der Diskurs des radikalen Konstruktivismus. Suhrkamp, Frankfurt a.M. 1996.

30. Haken, H. Die Selbstorganisation der Information in biologischen Systemen aus der Sicht der Synergetik. In: Küppers, B.O. (Hrsg) Ordnung aus dem Chaos. Piper, München, 3. Aufl., 1991

31. Sharov, A.A. Biosemiotics: A functional-evolutionary approach to the analysis of the sense of information. In: Seboek, T.A., Umiker-Seboek, J. (eds) Biosemiotics. Mouton de Gruyter, Berlin - New York 1992, S. 345-373.

32. Uexküll, Th. v., Wesiak, W. Wissenschaftstheorie: Ein bio-psycho-soziales Modell. In Adler, R.H., Herrmann, J.M., Köhle, K., Schonecke, O.W., v. Uexküll, Th., Wesiack, W (Hrsg) Psychosomatische Medizin. 5. Aufl., Urban \& Schwarzenberg, München - Wien Baltimore 1996, S. 13-50.

33. Erickson, M., Rossi, E. Hypnotherapie. Pfeiffer, München, 1981.

34. Gilligan, S. Therapeutische Trance. Das Prinzip der Kooperation in der Ericksonschen Hypnotherapie. Auer, Heidelberg, 1991.

35. Schmidt, G. "Wahrgebungen“ aus der „inneren" und "äußeren Welt" des Therapeuten und ihre Nutzung für zieldienliche therapeutische Kooperation. Familiendynamik 2000;2 177-205.

36. Revenstorf, D., Peter, B. (Hrsg) Hypnose in Psychotherapie, Psychosomatik und Medizin Springer, Berlin, Heidelberg, New York, Barcelona, Hongkong, London, Mailand, Paris, Singapur, Tokio, 2001

37. Ross, U.H. Tinnitus - So finden Sie wieder Ruhe. Gräfe \& Unzer, München, 2006.

38. Rainville, P., Hofbauer, R., Bushnell M.C., Duncan, G.H., Price, D.D. Hypnosis modulates activity in brain structures involved in the reg ulation of consciousness. J Cogn Neurosci 2002;14:887-901.

39. Rainville, P., Hofbauer, R.K., Paus, T., Duncan, G.H., Bushnell, M.C., Price, D.D. Cerebra mechanisms of hypnotic induction and sug gestion. J Cogn Neurosci 11:110-125, 1999.

40. Gutscher, H., Hornung, R., Flury-Kleubler, P. Das Transaktionspotentialmodell: Eine Brücke zwischen salutogenetischer und pathogenetischer Sichtweise. In: Margraf, J., Siegrist, J. Neumer, S. (Hrsg) Gesundheits- oder Krankheitstheorie? Saluto- versus pathogenetische Ansätze im Gesundheitswesen. Springer, Berlin, Heidelberg, New York 1998, S. 49-70.

41. Lang, J., Wachsmuth, W. Übergeordnete Systeme. In: J. Lang \& W. Wachsmuth (Hrsg): Praktische Anatomie. Bd. I, Teil 1: Kopf, Teil A Springer, Berlin 1985, S. 491.

\section{Korrespondenzadresse:}

PD Dr. med. Uwe H. Ross

Arzt für HNO-Heilkunde - Psychotherapie Hypno-systemische Beratung und Therapie Luisenstrasse 6, DE-79098 Freiburg

Dr-Ross@web.de

www.per-sono.de 